\title{
Significance of relationships in the cognitive apprenticeship of medical specialty training
}

\author{
A A Khine, ${ }_{1}^{1}$ MMed (Chemical Pathology), MPhil (Health Sciences Education); N Hartman, ${ }^{2} \mathrm{PhD}$ \\ ${ }^{1}$ Division of Chemical Pathology, Department of Pathology, Faculty of Medicine and Health Sciences, National Health Laboratory Service, \\ Stellenbosch University, Cape Town, South Africa \\ ${ }^{2}$ Department of Health Sciences Education, Faculty of Health Sciences, University of Cape Town, South Africa
}

Corresponding author: A A Khine (ayeaye.khine@nhls.ac.za)

\begin{abstract}
Background. The cognitive apprenticeship model is universally recommended for medical specialty training and has been introduced in some clinical disciplines by consultant specialists through the personal coaching of students and participation in the community of practice. In post-apartheid South Africa (SA), transformative initiatives gave rise to significant numbers of students from disadvantaged backgrounds in higher education that led to racial and sociocultural diversity among students and their consultants. Most notably, this occurred in medical specialties, where the number of students is much smaller than in undergraduate medicine. This stimulated interest in how this landscape may influence the cognitive apprenticeship model.

Objectives. To explore how former students of a medical specialty discipline conceived the nature of racial and sociocultural diversity in their learning environment and if/how this influenced their relationships with peers and consultants.

Methods. A qualitative enquiry was conducted with 9 formal postgraduate students (registrars) from 6 universities in SA. Data collection was through in-depth individual interviews with open, semi-structured questions. Data were analysed, recognising sub-themes and themes, and interpretation was done in a social constructionist approach of epistemology, where the participants and researcher co-construct the concepts.

Results. Participants conceived the sociocultural diversity as personal differences and related their experience of not receiving one-on-one mediation or mentoring to a lack of relationship with the consultants, which was believed to be underpinned by sociocultural differences. Power-plays in departmental culture also inhibited the legitimate access and participation of postgraduate registrars in the community of practice, inhibiting their growth of professional expertise.

Conclusions. Cognitive apprenticeship in medical specialty training has specific challenges in the context where postgraduate students and consultants are from societies previously divided by inequalities. Common acknowledgement was that learning in collaboration begins with learning to know each other and by forming relationships. Students faced challenges seeking professional mentorship, which was conceived as a principal contributing factor in their failure to learn.
\end{abstract}

Afr J Health Professions Educ 2021;13(1):36-40. https://doi.org/10.7196/AJHPE.2021.v13i1.1114

The approach to education for medical specialties has universally been postulated as cognitive apprenticeship training to develop novices into experts. This model requires mediation by a mentor, with participation in the community of practice $(\mathrm{CoP})$ and learning through situated contexts, each of which places importance on meaningful engagement and a close relationship between the educator and the student. ${ }^{[1-4]}$

The study was set in the context of formal postgraduate students who were training in one of the medical specialty programmes in pathology from 2006 to 2012, offered at 6 medical universities across South Africa (SA). The training programme was $4-6$ years and students wrote the national exit examination managed by the Colleges of Medicine of South Africa (CMSA). The students, also referred to as registrars, are employed by the service provider as trainees while enrolled with academic departments, and learn from consultants in the same department, who are experts in the field. With cognitive apprenticeship training in this discipline, consultants are the trainers who facilitate registrars' cognitive development through personal mentoring, using situated learning opportunities and encouraging them to participate in the departmental CoP, which is referred to as legitimate peripheral participation. ${ }^{[5]}$ This requires mediating one's development in the zone where the registrar moves from the previous or current knowledge and capability to another level, closer to the expertise in the discipline mediated by the mentor. Far more learning opportunities are provided through informal discussions with peers and consultants when discussing real-life problems occurring during daily service and practice than in a formal setting. Learning and development can only be realised in such situations if there is an active engagement between the parties. The need to engage and interact closely places relationships at the centre of the training programme. In some medical specialties, the number of students and consultants is very limited. With the recent dawn of transformation, increased numbers of black students entered medical specialties where most of the consultants were whites and Indians. Given the landscape of historical divisions with inequalities in SA, developing relationships of learning parties in this context can be challenging.

\section{Objectives}

We set out to explore how former postgraduate students conceived the racial and sociocultural diversity in their learning environment; and if, and how, these influenced their relationships with their consultants and peers, and ultimately their learning. Henceforth, registrars are referred to as participants. 


\section{Methods}

The study employed a qualitative enquiry to attempt to unpack the participants' conceptions around racial and sociocultural diversity of the relevant medical specialty in their learning environment; and if, and how, this influenced the relationships among learning parties.

\section{Racial profile of participants}

The sample consisted of postgraduate registrars who were formally in the training programme between 2006 and 2012 at 6 universities in SA. There was a total of 12 registrars, of whom 9 agreed to participate. Five of the 9 left the specialty programme unqualified and 4 left as qualified specialists. The researcher's own department was excluded for ethical purposes.

Of the 5 who left the programme unqualified, 3 were black South Africans, 1 was an SA Indian, and the other was an Indian childhood immigrant to SA. In the group that qualified, 3 were SA blacks and 1 was an SA Indian. Gender is not described, as participants used pseudonyms across gender for anonymity. In both groups, participants were trained in their respective departments by consultants who were in the majority white (Afrikaans or English speaking) and SA Indians, with 1 SA black consultant and 1 foreign consultant.

\section{Data collection and analysis}

Participants were interviewed by the researcher/author individually and discretely after obtaining informed consent, agreeing on using self-selected pseudonyms and gender, and keeping the identity of the university of study hidden. Participants agreed to disclose their racial identity, as it was crucial information for the purposes of the study. The interviews for each participant were conducted at their chosen place and time, using a standard tool that was approved by the departmental technical committee (Department of Health Sciences Education, University of Cape Town) and the ethics committee. It consisted of semi-structured, open-ended questions that probed the landscape of the learning environment and relationships, with impact on the training and learning (the questions are available from the corresponding author on request). Interviews were audio-recorded by the researcher, transcribed by the professional transcriber and coded by the researcher, using deductive and inductive approaches based on the method of thematic analysis. ${ }^{[6]}$ Data were interpreted using a social constructionist approach. This method of epistemology was used to build knowledge from the concepts co-constructed by the students as peers through social interactions during their training; co-constructed with the researcher during the interviews; and co-constructed by an independent analyst during analysis.

An inductive coding approach provided systematic coverage of data, which was expected, based on the research questions and interview questions, and the deductive approach checked the data that emerged from interviews that were not initially expected. Using both at the same time ensured that all data had been covered. Together with the social constructionist epistemology, the trustworthiness of the data and interpretations were improved, as the latter affords multiple layers of perspectives with iterative processes of seeking consensus. The researcher was inevitably invested in this study, being one of the trainers in the discipline for many years, but her bias was reduced or controlled by self-awareness and reflexivity throughout the process of planning, interviews, data coding, analysis and interpretation. ${ }^{[7]}$ Individual participants received the transcript of their interviews and coding to ensure that their messages were captured correctly and important contributions were not missed in the coding. This is referred to as member checking and improved trustworthiness of the data. An independent co-analyst was employed for reviewing codes, patterns and interpretations. Data interpretation was also strengthened by comparing contributions from the participants from contrasting groups (the group that left the programme owing to failures and the group that qualified).

\section{Ethical approval}

Ethical approval for this study was obtained from the Human Research Ethics Committee, Faculty of Health Sciences, University of Cape Town (ref. no. HREC REF 656/2016).

\section{Results}

The interview questions probed into participants' history of learning (schooling and undergraduate study) as a foreground to the most recent postgraduate study in the relevant medical specialty, as well as family background and their concepts of sociocultural diversity. The participants, from low- to middlesocioeconomic family backgrounds, had lived through the country's apartheid system. Parents of all participants reportedly wished for their children to overcome inequalities and succeed in higher education. Some studied at single-race school; others had to adjust at multiracial schools and developed the skill of cross-cultural relationships. Nonetheless, they all enjoyed their schooling and fared well in matric. However, most struggled with a language barrier during undergraduate medical studies and faced challenges with social and cultural diversities, which they interpreted as differences. As SA blacks and Indians, they experienced being underrated by white consultants during undergraduate medical training, which they felt became more pronounced during postgraduate training owing to fewer students and working closer with consultants. In the following sections, participants are cited (two groups), where their contributions are quoted: the group that qualified and the group that left the training programme unqualified.

\section{Relationships in the learning environment}

The relationship between the participants and their consultants was conceived as multiple layers: as student and mentor, student and supervisor in research projects, junior and senior members in the CoP, and work colleagues in the same department. Most participants reported being treated differently by consultants because of their race, and sociocultural and economic status. The striking phenomenon is that all participants, including those who successfully qualified, experienced these differences; they had relationship issues with consultants; and they constructed that their learning was negatively affected, although 4 managed to navigate these difficulties and succeed.

Participants expressed the need to be recognised as professionals, given that they were already qualified doctors with some practice experience. This perception came from the conjecture by their consultants that they were not academically prepared or smart enough to be successful in the course, as a participant reflected: '... we're labelled as useless people and people who can't learn anything, so, it broke down our relationships in that respect.' There was a disjointed expectation between consultants and registrars across both groups. The consultants believed that at a postgraduate level a registrar should self-study and initiate enquiry or ask if they needed assistance. However, the participants felt that they needed consultants to initiate regular supervision and professional mentoring, as highlighted by a participant from the qualified group: 'It is also important to be guided because the consultants have the experience and they've got 
knowledge that you're still trying to find out, so they would be able to guide you better.' Another qualified participant mentioned: 'The former peers were assuming that there's continuous learning. They thought there would be continuous contact with a consultant. They realised there wasn't much contact, it was self-learning, and they left the programme.'

A lack of contact sessions or mediation from consultants led to participants constructing that the consultants had no interest in their learning process, as one participant recalled: '... there's no consultant that sat with you and looked at results with you, and said, look at this, look at this. One consultant did it initially, but it faded away. It was very brief, and it was rather interrogating. I want to show you that you're not doing it properly type of attitude. So, it was a very interrogating approach.' The differences in opinion and expectations created tensions, disconnection with consultants and unhappiness of participants, which persisted throughout the 4 - 6 years of their training (in the group that left). They felt that they could not solve this disconnection owing to a lack of relationships. A participant in this group highlighted that: 'We had no relationships, and that was identified as a shortcoming in our training.'

The lack of or strained relationships were also due to restricted contact time with consultants who focused on their own professional growth or agendas, as mentioned by participants. Furthermore, participants from both groups felt that initiating contact or approaching consultants was difficult, citing feelings of discomfort that made the interactions with consultants from across cultures challenging. Both groups experienced apparent powerplay within their respective departments among the consultants or between the consultant and the head of department (HOD). This contributed to difficulties experienced by the registrars in approaching one consultant over the other, as a participant who left the programme identified: '... there was no coherence in the department amongst the [head of department] HOD and consultants, it was more like personalities and power struggles.

Interestingly, despite sharing these challenges, participants in the qualified group worked out ways to approach and engage with at least one consultant, either in their department or from the private sector. They also managed to form relationships with peers and consultants from other universities in the same field. This showed that relationships were formed more easily when dealing with consultants from another institution, as there was no history of tension or grudges or preformed ideas against each other.

One of the qualified participants advised, '... if somebody feels that it's not their responsibility to teach you, that's how they feel about it. There's nothing they're going to do. You have to find a way around.' This participant had worked out that the culture of the department could be navigated. He or she tapped into a consultant's expertise by initiating enquiry and bringing problems identified during daily service provision to the consultant, with possible solutions.

Another qualified participant had a different experience, whereby he or she was personally mentored by the HOD and had regular formative assessments early on in the training. This participant conceived that the success in forming a relationship with the HOD was due to his or her upbringing rooted in showing respect to elders and co-operating with their suggestions. Another qualified participant, having been unsuccessful in many attempts, recollected how he or she was noticed by the consultant from the private sector, who was also the external examiner at an exit examination. The consultant examiner noticed the participant's good behaviour and potential during the examination and offered private mentoring. The participant believed it was his or her obedience and non-confrontational attitude that won the heart of the consultant. At the next exit examination, the participant passed and qualified, and went on to join the mentor in a private business.

\section{Curriculum, training programme and behavioural factors}

Apart from the conceptions around relationships, there was other important information that emerged from the data, such as lack of awareness regarding the scope, depth and outcomes of the curriculum during the time of training. Lack of awareness was not only applicable to registrars, but also to consultants, as the blueprint of the curriculum was only completely mapped out in 2013 for this discipline, and for many other medical specialties it may still be pending. From the contributions, it was noted that the formal training programme at the various departments was highly variable and not all departmental programmes were aligned to what was required for registrars to know before sitting the exit examination. Personal mentoring, mediation and participation in the CoP, as suggested and mapped out in the cognitive apprenticeship model, did not exist, according to the participants.

Participants reported a scarcity of opportunities for informal interactions and learning in situatedness that had prevented their development. One participant, an ethnic African-language speaker and second-language English speaker, believed that this was due to a language barrier, with consultants and peers speaking Afrikaans during informal discussions: 'I now was exposed in an Afrikaans environment ... although English was there, it was predominantly Afrikaans. I was thrown completely out of my culture. Well, mainly it was through the language used during formal or informal learning sessions. Language sort of over-ruled or dominated us by exclusion so we were already disadvantaged. The rest is taken after the fact that now we were lost in their discussion. So, we appeared as if we did not know anything - then we became outsiders or less well prepared. Another participant related: 'It was a huge shocker. Not necessarily because it was a different language, but the fact was the expectation was that I had to know Afrikaans for me to learn medicine.' A participant reported being too slow in answering the consultant's questions after having to translate into her home language, which made her feel uncomfortable to join discussions: 'I talked slowly as I had to translate English to vernacular and back there again and my white peers would answer the questions much faster as it was their mother tongue.' Such incidences hampered participants' access to situated learning and participation in the CoP.

As participants from the qualified group provided their recommendations, self-directed learning (SDL) was one of the contributory factors in their success, but with the caution that they needed considerable guidance from the consultants. Their construction of SDL was to find alternative ways to reach out and tap into the expertise of at least one consultant who was knowledgeable and skilful in practice. Two participants in the qualified group took the initiative in identifying problems and finding solutions, and approached a consultant for further discussion, as one of them expressed: '... you need the consultants to guide you but most of the effort has to come from you, so that at least when you approach them [consultants] with your case, you know that from your side you've done your part. I think it would be embarrassing to ask them and you don't know anything in the background, so better gather the information.

Furthermore, there were behavioural expectations from the consultants, i.e. submissiveness and obedience, reported by both groups. However, registrars in the group that left felt that they should have maintained being assertive, whereas those in the group that qualified believed in adjusting to 
what was expected by the consultants - at least for a period of the training. Participants in both groups believed that these demands were underpinned by power and control issues from the consultants, which strained the relationships.

The findings highlight that the feelings of sociocultural differences, assumptions rooted in these differences, choice of language use, especially in the informal discussions, disjointed expectations, as well as behaviour diversity, are intricately related to strained relationships and ultimately to loss of learning opportunities.

\section{Discussion}

The social domain of the cognitive apprenticeship model plays a pivotal role in social and cultural engagement between the registrar and consultant for facilitating learning and development into professions. ${ }^{[1]}$ This promotes internalisation of the discipline-specific culture and skills to a level closer towards expertise, thus preparing registrars for the expected outcomes. In this model, coaching by the expert is related strongly to the mediation in the zone of proximal development by Vygotsky, ${ }^{[8]}$ which requires the mentor to know and understand the weaknesses and strengths of the novice registrar to grow in their potential. Learning in situated contexts can only occur if students participate in the problem-solving processes and contributions in the CoP. Through participation and mediation, the cultures of the discipline and attitude of the profession are transferred.

Responding to the difficulties expressed, qualified participants had relied heavily on SDL and peer learning, given the scarcity of opportunities to learn from their senior consultants. Realising that they needed a consultant to guide them with SDL, they managed to navigate the power-plays in their departmental culture and initiate engagement with at least one consultant who was impressed with their persistence. This experience agrees with the notion of SDL that recently emerged as requiring guidance from more knowledgeable others in a facilitative environment, as shown in the recent literature, where one of the articles is a meta-analysis review. ${ }^{[5,9,10]}$ Qualified participants had realised that it is possible to adjust to what is expected of them, at least for the period of their training, to be accepted by the CoP to improve their chances of success.

In the eyes of participants who left the programme unsuccessfully, the lack of day-to-day supervision and coaching by not having a mentoring relationship led to their failure to progress in their studies. Cruess et al. ${ }^{[2]} \mathrm{claim}$ that the attributes of the trainer as an effective role model are compassion, honesty and integrity. Equally important is an ability to maintain interpersonal relationships, showing enthusiasm for practice and teaching, and an unwavering effort to thrive for excellence. The authors cautioned that institutions tend to accept situations where clinical staff members are overworked, which leads to insufficient time for coaching and mentoring. A lack of institutional support for such activities also contributes to students and clinical teachers failing to form relationships.

Vygotsky ${ }^{3]}{ }^{[8]}$ concept of mediation focuses on the sociocognitive process of conceptual development, although there is no emphasis on the personal differences between mentor and mentee. Another theorist, Bandura, ${ }^{[11]}$ stressed the importance of a continuous interaction between people in the learning environment, their behaviours and personal factors, as well as their cognition. He referred to this interactive environment as a reciprocal causation model highlighting their effects on one another.

Lave and Wenger ${ }^{[12,13]}$ explain the effects of power relations within the $\mathrm{CoP}$ and how these impact on participation and contributions. Registrars and consultants work together as colleagues on a daily basis, and their social interactions constitute learning in situatedness and problem solving that are critical for professional development, culture and attitudes of the profession, and the road map towards expertise of the discipline. Lack of understanding, encouragement or mediation can lead to feelings of alienation. Bezuidenhout et al., ${ }^{[3]}$ in their SA study, reported alienation and lack of engagement perceived by novice registrars in a postgraduate education programme. The authors constructed that the source of feeling alienated could be due to the lack of relationships that students expected to experience, but did not get an opportunity to develop.

Relationships have emerged as being significant in the development of participants in this study. This relates to the theory of the human development concept that mental capacity and a sense of self-confidence develop due to the continuous process of communication and relationship formation. ${ }^{[14]}$ Relationships are vehicles for learning to develop cognitively, provide opportunities for student and teacher to engage by sharing conversations and experiences, and understanding each other's cultural identities, which may allow both parties to navigate training and learning in the complex social environment. ${ }^{[15]}$

Bradbury ${ }^{[16]}$ stressed that the mentor should understand and respect the level of development of the novice, evaluate their beliefs regarding learning, recognise the significance of their prior learning and contribution as a source of knowledge, and use the expertise of self and student in developing new ideas. This reflects reciprocity and interdependence in their relationship. Findings in this study, together with the literature, show the importance of co-operative learning that equips both consultant and registrar for work-place collaboration, fostering relationships and cultivating initiatives in enquiry in medical specialties. Reflecting on these theories illuminates how, in this study, the lack of relationships influenced learning negatively, while it also assisted in explaining how the few participants that were mentored, secured their learning and succeeded through their relationships with their mentors.

\section{Reflections}

At the protocol stage and during data collection, race was used as a major construct, as it is in SA's higher education arena. It was therefore relevant to be included in this study. However, data revealed that there were other factors that influenced relationships, such as personalities. This was more apparent during discussions with participants from the qualified group, who constructed race as not being a sole factor for the lack of relationships. Thus, the study was framed as a doubled-research approach: firstly, the study problem with a strong racial construct, and then it considered interpretations towards deracialising, rather than the mid-stream method described by Muzzin and Mickleborough. ${ }^{[17]}$

\section{Study limitations}

Nine of 12 participants were registered during 2006 - 2012 for the discipline of pathology in SA. Although the sample size may seem small, it comprised $75 \%$ participation as purposeful sampling. The study excluded consultants of the same departments to protect identities of participants, and therefore could not triangulate the data from their perspectives. A further study is recommended to interview the consultants. Moreover, the findings may only be relevant for the abovementioned training period, as the curriculum was established in 2013 and in some departments training programmes might have been updated. As it has been a number 
of years since the study was conducted and much progress has been made in terms of decolonising the curriculum, as well as improving racial relationships, it is hoped that current students are not experiencing the dynamics described in this article.

\section{Conclusion}

In the context of post-apartheid transformation in SA, students in medical specialties from a previously disadvantaged background may perceive that divisions are still apparent in their training. When students immerse in an environment where they perceive they are treated differently, relationships can be threatened. Lack of relationships with mentors can have a negative impact on learning and professional development, as the opportunities for cognitive apprenticeship are lost. Providing workshops with activities towards cross-cultural immersion should be part of training for consultants and registrars to foster relationships. A mentor should be identified for each registrar at the time of joining the programme. The number of registrars per mentor consultant should be established by the Health Professions Council of SA and the employer, and they should be aware of the importance of relationships in medical specialty training to ensure the success of all trainees.

\section{Declaration. None.}

Acknowledgements. We thank the study participants for their valuable contributions.

Author contributions. AAK collected and analysed the data and drafted the manuscript and $\mathrm{NH}$ made inputs.
Funding. None.

Conflicts of interest. None.

1. Collins A. Cognitive apprenticeship. In: Sawyer RK, ed. The Cambridge Handbook of the Learning Sciences Cambridge: Cambridge University Press, 2006:47-60.

2. Cruess SR, Cruess RL, Steinert Y. Role modelling: Making the most of a powerful teaching strategy. BM 2008;336:718-721. https://doi.org/10.1136/bmj.39503.757847.BE

3. Bezuidenhout J, Cilliers F, van Heusden M, Wasserman E, Burch V. Alienation and engagement in postgraduate training at a South African medical school. Med Teach 2011;33(3):e145-e153. https://doi.org/10.3109/014215 9X.2011.543198

4. Genzen JR, Krasowski MD. Resident training in clinical chemistry. Clin Lab Med 2007;27(2):343-358. https://doi org/10.1016/j.cll.2007.03.007

5. Couper I, Town C. Self-directed learning in educating health professionals: A scoping review of the literature. Teaching Advancement at University (Tau) Fellowships Programme. 2016. http://heltasa.org .za/wp-content/ uploads/2016/10/TAU-PROJECT-report-rev-Ian-Couper-FINAL.pdf (accessed 24 November 2020).

6. Braun V, Clarke V. Thematic analysis. In: Cooper H, ed. The APA Handbook of Research Methods in Psychology, Vol. 2. Research Designs. Washington, DC: American Psychological Association, 2012:57-91.

7. Lincoln Y, Guba E. Ethics: The failure of positivist science. Rev High Educ 1989;12(3):221-240. https://doi Lincoln Y, Guba E. Ethics:
org/10.1353/rhe.1989.0017

org/10.1353/rhe.1989.0017
8. Vygotsky LS. Interaction between learning and development. In: Mind and Society. Cambridge, MA: Harvard University Press, 1978:79-91.

9. Knowles MS. Self-directed Learning: A Guide for Learners and Teachers. New York: Association Press, 1975.

10. Bok HG, Teunissen PW, Favier RP, et al. Programmatic assessment of competency-based workplace learning: When theory meets practice. BMC Med Educ 2013;13:1-10. https://doi.org/10.1186/1472-6920-13-123

11. Bandura A. Social cognitive theory. In: Vasta R, ed. Annals of Child Development. Vol. 6. Greenwich, CT JAI Press, 1989:1-60.

12. Lave J, Wenger E. Situated learning: Legitimate peripheral participation. In: Pea R, Brown JS, eds. Learning in Doing Cambridge, MA: Cambridge University Press, 1991:95. https://doi.org/10.1017/CBO9780511815355

13. Wenger E. Communities of practice and social learning systems: The career of a concept. In: Blackmore C, ed. Socia Learning Systems and Communities of Practice. Berlin: Springer Verlag and Milton Keynes: Open University, 2010. 4. Fogel A. Developing Through Relationships. Chicago: University of Chicago Press, 1993.

15. Goodman S. The importance of teaching through relationships. 2015. https://www.edutopia.org/blog/importanceteaching-through-relationships-stacey-goodman (accessed 24 November 2020).

16. Bradbury LU. Educative mentoring: Promoting reform-based science teaching through mentoring relationships. Bradbury LU. Educative mentoring: Promoting reform-based

17. Muzzin L, Mickleborough T. What does 'race' have to do with medical education research? Med Educ 2013;47:760-767. https://doi.org/10.1111/medu.12186

Accepted 16 January 2020 